\title{
Neuroticism, Smoking, and the Risk of Parkinson's Disease
}

\author{
Johanna Sieurin ${ }^{\mathrm{a}}$, Yiqiang Zhan ${ }^{\mathrm{b}, \mathrm{c}, \mathrm{d}, *}$, Nancy L. Pedersen ${ }^{\mathrm{a}}$ and Karin Wirdefeldt ${ }^{\mathrm{a}}$ \\ ${ }^{a}$ Department of Medical Epidemiology and Biostatistics, Karolinska Institutet, Stockholm, Sweden \\ ${ }^{\mathrm{b}}$ School of Public Health, Sun Yat-sen University, Shenzhen, China \\ ${ }^{\mathrm{c}}$ Institute of Environmental Medicine, Karolinska Institutet, Stockholm, Sweden \\ ${ }^{\mathrm{d}}$ German Center for Neurodegenerative Diseases, Ulm Germany
}

Accepted 22 April 2021

Pre-press 15 May 2021

\begin{abstract}
.
Background: The relationship among neuroticism, smoking, and Parkinson's disease (PD) is less examined.

Objective: To examine the causal associations between neuroticism, smoking initiation, and the risk of PD.

Methods: We performed a two-sample Mendelian randomization (MR) design in a network framework. Summary statistics from meta-analyses of genome-wide association studies (GWAS) were based on large cohorts of European ancestry. Study participants were from various cohort studies for neuroticism and smoking initiation, and case-control studies or cohort studies of PD from previously published GWAS meta-analyses. Patients with PD were ascertained from either clinical visit or self-reported.

Results: The two-sample MR analysis showed no evidence for a causal association between neuroticism and PD risk (odds ratio [OR] $0.86,95 \%$ confidence intervals [CIs] 0.67 to 1.12 ). While we did not find a significant association between neuroticism and PD, one SNP, rs58879558 (located in MAPT region), was associated with both neuroticism and PD. We found a significant association of neuroticism on smoking initiation (OR: 1.10, 95\% CI: 1.05 to 1.14). Further, our results provided evidence for a protective effect of smoking initiation on the risk of PD (OR: $0.75,95 \% \mathrm{CI}$ : 0.62 to 0.91 ).

Conclusion: These findings do not support a causal association of neuroticism on PD risk. However, they provide evidence for a causal relationship between neuroticism and smoking initiation and a strong causal effect of smoking initiation on a reduced risk of PD.
\end{abstract}

Keywords: Neuroticism, smoking, Parkinson's disease, mendelian randomization.

\section{INTRODUCTION}

Parkinson's disease (PD) is the second most common neurodegenerative disease and the most prevalent movement disorder. The vast majority of PD cases are idiopathic, caused by an interplay of genetic, environmental and lifestyle factors [1]. The hypothesis that PD is associated with a specific personality

${ }^{*}$ Correspondence to: Yiqiang Zhan, Institute of Environmental Medicine, Karolinska Institutet, Stockholm, Sweden. Tel.: +46 0 8524822 89; E-mail: yiqiang.Zhan@ki.se. type is over a century old and supported by several observational studies [2, 3]. It has also been suggested that certain personality traits are present before PD diagnosis and represent risk factors or a prodromal stage of $\mathrm{PD}$, but few longitudinal studies have been performed. In two studies with up to four decades-long follow-up times, the personality trait neuroticism was found to precede PD diagnosis [4, 5]. Neuroticism is a personality trait characterized by an increased tendency to experience negative emotions such as anxiety, sadness, and fear, is relatively 


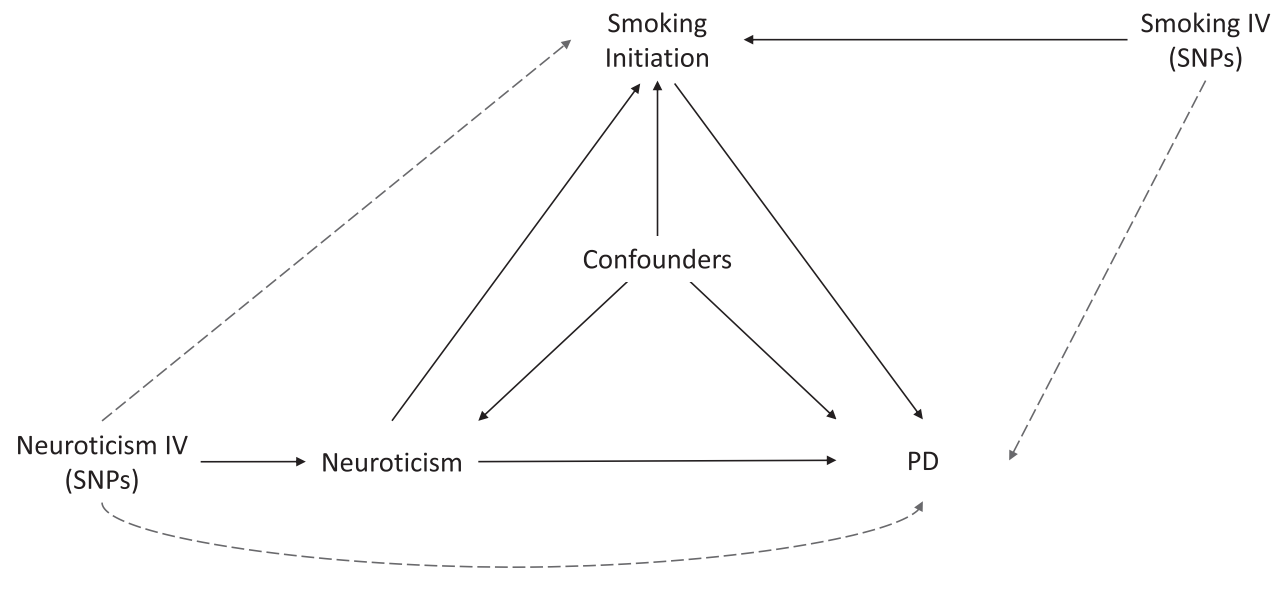

moking IV

SNPS)

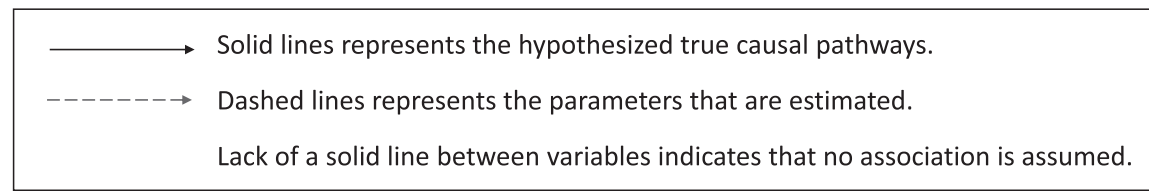

Fig. 1. Illustration of study design and instrumental variable assumptions underlying Mendelian randomization. In this study, SNPs robustly associated with an exposure are used as instrumental variables to assess the effect of an exposure on an outcome (dashed lines), which equals the product of multiplying the potential true effects (represented by solid lines) mediating this estimated association. This figure also illustrates the assumptions underlying a Mendelian randomization study. 1) The genetic variants are associated with the exposure, which were tested by previous GWAS studies. 2) The genetic variants are not associated with confounders of the exposure-outcome associations (lack of solid lines). 3) The only effect of the genetic variants on the outcome must be through the exposure, and not through any direct or alternative pathways (lack of solid lines). IV, instrumental variable; SNP, single nucleotide polymorphism; PD, Parkinson's disease

stable throughout life and has a substantial genetic component [6-8]. Available evidence indicates that there is no genetic correlation between neuroticism and PD [9].

There is a well-documented association between neuroticism and increased smoking behaviours from observational studies $[10,11]$ that was suggested to be causal in a recent Mendelian randomization (MR) study, although the results were not completely conclusive [12]. Further, there is an overwhelming amount of evidence from observational studies showing that smoking is associated with a reduced risk of $\mathrm{PD}$ and there is an ongoing debate on how to interpret these findings [13-15]. Results from observational studies analysing the effect of parental smoking as an instrumental variable (IV) or passive smoking on PD risk indicate that the association might be causal $[16,17]$. Several alternative theories have been proposed, including confounding by a low-risktaking personality or reverse causation because of a decreased responsiveness to nicotine during the prodromal phase of PD, making it easier to quit smoking $[18,19]$. Nevertheless, smoking may act as a mediator that suppresses the observed effects of neuroticism on PD [4].
Results from observational studies are prone to confounding, which makes it difficult to establish a causal relationship, particularly for PD which has a late onset and a long prodromal period [20]. Therefore, it is largely unknown whether neuroticism is causal risk or protective factors for PD, or if they represent a preclinical phase of PD. MR is an approach that can control for confounding by using genetic variants as probes for an exposure of interest. Provided that the underlying assumptions hold, inference about causality can be made. In this study, we used two-sample MR methods to disentangle the causal associations between neuroticism, smoking, and PD.

\section{MATERIALS AND METHODS}

\section{Study design}

We conducted a two-sample MR study using summary-level statistics from previously published genome-wide association studies (GWAS) in a network framework. Three main analyses were performed to explore causal associations between (I) neuroticism and PD, (II) neuroticism and smoking initiation, and (III) smoking initiation and PD (Fig. 1). 


\section{Data sets}

Single nucleoid polymorphisms (SNPs)

associated with neuroticism

We extracted summary statistics for genetic variants influencing neuroticism levels identified from a recent GWAS meta-analysis on neuroticism [9]. This study reported 136 independent genome-wide significant loci $\left(p<5 \times 10^{-8}\right)$ in a sample of 449,484 individuals of European descent, comprised of data from the UK Biobank (UKB) $(n=372,903)$ [21], 23andMe, Inc. $(n=59,206)$ [22], and Genetics of personality consortium (GPC) $(n=17,375)$ [23]. In the current study, we used summary statistics based on analyses excluding 23andMe data, as these summary statistics were publicly available and have no sample overlap in the two-sample MR analyses. Information on neuroticism was obtained through digital questionnaires in all samples: 12 yes/no items of the Eysenck Personality Questionnaire-Revised Short form (EPQ-RS) for UKB and 12 five-point Likert-scale items from the Neuroticism Extraversion Openness-Five Factor Inventory (NEO-FFI) for GPC.

\section{SNPs associated with smoking initiation}

We used publicly available summary statistics from a recent GWAS meta-analysis of smoking behaviors to extract summary statistics of genetic variants influencing smoking initiation. This is a binary phenotype defined as having ever been a regular smoker or not. The study reported 378 independent genome-wide significant loci $\left(p<5 \times 10^{-8}\right)$ in a sample of 1,232,091 individuals of European descent [24].

\section{PD GWAS}

We extracted summary statistics of associations between identified SNPs and PD from a large GWAS meta-analysis. Datasets included in this metaanalysis were the same as in a large recent PD GWAS meta-analysis [9] excluding proxy PD-case data from the UK Bio Bank and a smaller sample from the System Genomics of Parkinson's Disease (SGPD) whose data were not shared publicly. The data included 23and Me PD case-control summary statistics, which were provided by 23 and Me under an agreement with 23 and Me that protects the privacy of the 23 and Me participants. This resulted in GWAS summary statistics data for 36,752 PD cases and 929,806 controls of European ancestry.

Separate GWAS meta-analyses of PD were performed dependent on definition of PD used (clinical diagnosis or self-reported). This resulted in a GWAS meta-analysis involving 9,157 self-reported PD cases and 822,855 controls (from 23andMe), and a GWAS meta-analysis involving 27, 595 clinical diagnosis PD cases and 106, 951 controls (all other datasets included in the meta-analysis).

\section{Data extraction and harmonization}

After extracting GWAS summary statistics (i.e., effect allele, non-effect allele, beta value/log odds ratio, standard error, and $p$-value) for each identified SNP influencing the exposure of interest (neuroticism or smoking initiation), the summary statistics for the same SNPs were extracted from the GWAS dataset of the outcome of interest (PD or smoking initiation). If the SNPs were unavailable in any of the exposure/outcome GWAS datasets, or if the SNPs were palindromic (i.e. A/T or $\mathrm{G} / \mathrm{C}$ ), proxy SNPs that were not palindromic and with an $r^{2}>0.8$ were identified using LDlink [25]. The SNP-exposure and SNP-outcome associations were then harmonized to ensure that the direction of effects reflected the same alleles.

\section{Estimation of causal effects}

We used the inverse variance weighting (IVW) method as the main method to estimate the causal effects. The IVW estimate combines the ratio estimate from multiple genetic variants and can be viewed as a weighted average of ratio estimates of genetic effects on outcome and exposure for each SNP [26]. Additional MR methods that allow for different types of genetic pleiotropy and rely on partly different assumptions were performed to check the robustness of our findings. The MR-Egger regression [27] provides less biased effect estimates in the presence of directional pleiotropy and heterogeneity. This is achieved by allowing an intercept term, unlike the IVW method, which represents the average pleiotropic effects of the SNPs. The beta coefficient of the slope from MR-Egger regression may provide a valid causal estimate even though the SNPs have pleiotropic effects on the outcome, if they are not proportional to the SNP-exposure effect. However, MR-Egger is more sensitive to associations between SNPs and exposure-outcome confounders and it is less powerful than the IVW method. We further performed analyses using the weighted median method [28], which uses the weighted median of the ratios of association with outcome and exposure for all SNPs. 
The weighted median method may provide robust estimates even though some of the SNPs are not valid instrumental variables as long as at least $50 \%$ of the weights come from valid SNPs.

\section{Sensitivity analyses}

We plotted the effect estimates with exposure and outcome for each SNP in order to visually identify any outliers. Identified outliers with a potential pleiotropic effect on outcome were removed from the main analyses. We repeated the analyses by excluding the use of proxy SNPs. We also performed separate MR analyses using different definitions of $\mathrm{PD}$ (clinical diagnosis vs self-reported). The $p$-value of Cochran's Q statistic was used to test for heterogeneity, whereas the $\mathrm{I}^{2}$ statistic was used to estimate the degree of heterogeneity. A significant intercept parameter from the MR-Egger regression was used as evidence for directional pleiotropy [27]. We also assessed the probable directional pleiotropy using a funnel plot similar to that which is used to assess for publication bias in meta-analysis. A leave-one-out analysis was conducted by removing a single SNP in turn from the analysis for each of three associations. The fluctuation of the estimates in response to excluding each genetic variant reflects the possibility of outlier genetic variant in the estimation.

We calculated power for the MR analysis of neuroticism on PD using an online calculator (https:// shiny.cnsgenomics.com/mRnd/). By assuming the odds ratio per standard deviation increase of neuroticism score was 1.15 with variance explained by IVs being $1.3 \%$, the power of current MR analysis was 91\%. All analyses were performed in R 3.6.1. We used the MendelianRandomization package [29] to perform the MR analyses.

\section{Ethical approval}

This study was approved by the Regional Ethical Board in Stockholm. This study only used summary level statistics and no individual level data were used. Informed consent was not required.

\section{Data availability}

The GWAS summary statistics can be obtained from the 23andMe research team under a data transfer agreement.

\section{RESULTS}

\section{Neuroticism and PD risk}

Among the 136 neuroticism SNPs, 29 were unavailable in the PD GWAS and/or palindromic. We were able to identify 22 proxy SNPs leaving 129 SNPs for analyses (Supplementary Table 1). MR analyses showed that neuroticism was associated with a lower risk of PD (odds ratio [OR]: 0.72, $95 \%$ confidence interval [CI]: 0.52 to 1.00 ). However, the intercept from MR-Egger regression was statistically different from the null $(\beta=0.03,95 \% \mathrm{CI}$ : 0.01 to 0.06 ), implying that there might be horizontal pleiotropy for these SNPs. Furthermore, the Cochran's Q test for heterogeneity was significant ( $p$ value $=0.00$ ) with an $\mathrm{I}^{2}$ of $20 \%$. Further, the weighted median estimate was in the same direction, albeit with a wider confidence interval (Table 1). We plotted the effect estimates on neuroticism and PD for each SNP to visually screen potential outliers (Fig. 2). The SNP rs58879558 (located in MAPT region, a proxy for rs77804065, $r^{2}=1.0$ ) was identified as an outlier with a potential pleiotropic effect as it was significantly associated with PD. This SNP was also identified as an evident outlier in funnel plots used to assess directional pleiotropy (Supplementary Figures 1 and 2 ) and in the leave-one-out analysis (Supplementary Figure 3). As this may violate the MR assumptions, it was therefore removed. Reanalysis of the remaining 128 SNPs revealed no strong evidence for a causal effect of neuroticism on PD risk (OR: 0.86, 95\% CI: 0.67 to 1.12 ) (Table 1). The intercept from MR-Egger regression was not statistically significant, indicating that there was no evidence for horizontal pleiotropy for the remaining SNPs.

\section{Neuroticism and risk of smoking initiation}

We further examined whether neuroticism was causally related to smoking initiation. Among the 136 neuroticism SNPs, 24 were missing in the smoking GWAS and/or palindromic. We were able to identify 17 proxy SNPs leaving 129 SNPs for analyses (Supplementary Tables 1 and 2). The 129 SNPs used as instrumental variables for neuroticism provided evidence for a causal effect of neuroticism on an increased risk for smoking initiation using the IVW method (OR: 1.10, 95\% CI:1.05 to 1.14). Results were consistent with the weighted median method (OR: $1.10,95 \%$ CI: 1.06 to 1.15 ). The test of heterogeneity was significant, but the $\mathrm{I}^{2}$ of $0.0 \%$ indicated 
Table 1

Associations of neuroticism, smoking initiation, and Parkinson's disease using three Mendelian randomization methods

\begin{tabular}{|c|c|c|c|c|c|c|}
\hline \multirow{2}{*}{$\begin{array}{l}\text { Exposure - Outcome } \\
\text { Neuroticism - PD }\end{array}$} & \multirow{2}{*}{$\begin{array}{l}\text { \# SNPs } \\
129\end{array}$} & \multirow{2}{*}{$\begin{array}{l}\text { Method } \\
\text { IVW }\end{array}$} & \multirow{2}{*}{$\frac{\mathrm{OR}(\beta)^{*}}{0.72}$} & \multicolumn{2}{|c|}{$95 \% \mathrm{CI}$} & \multirow{2}{*}{$\frac{p}{0.05}$} \\
\hline & & & & 0.52 & 1.00 & \\
\hline & & Weighted Median & 0.92 & 0.74 & 1.15 & 0.48 \\
\hline & & MR-PRESSO & 0.73 & 0.50 & 1.05 & 0.15 \\
\hline & & MR Egger ${ }_{\text {slope }}$ & 0.09 & 0.02 & 0.44 & 0.00 \\
\hline & & MR Egger intercept $_{\text {t }}$ & $(0.03)$ & 0.01 & 0.06 & 0.01 \\
\hline \multirow[t]{5}{*}{ Neuroticism - PD } & $128 \dagger$ & IVW & 0.86 & 0.67 & 1.12 & 0.27 \\
\hline & & Weighted Median & 0.94 & 0.75 & 1.16 & 0.55 \\
\hline & & MR-PRESSO & 0.85 & 0.66 & 1.11 & 0.30 \\
\hline & & MR Egger ${ }_{\text {slope }}$ & 0.85 & 0.22 & 3.23 & 0.81 \\
\hline & & MR Egger intercept $_{\text {in }}$ & $(0.00)$ & -0.02 & 0.02 & 0.98 \\
\hline \multirow[t]{5}{*}{ Neuroticism - Smoking Initiation } & 129 & IVW & 1.10 & 1.05 & 1.14 & 0.00 \\
\hline & & Weighted Median & 1.10 & 1.06 & 1.15 & 0.00 \\
\hline & & MR-PRESSO & 1.10 & 1.05 & 1.13 & 0.00 \\
\hline & & MR Egger ${ }_{\text {slope }}$ & 0.99 & 0.81 & 1.20 & 0.91 \\
\hline & & MR Egger intercept $_{\text {t }}$ & $(0.00)$ & -0.00 & 0.01 & 0.29 \\
\hline \multirow[t]{5}{*}{ Smoking Initiation - PD } & 365 & IVW & 0.75 & 0.62 & 0.91 & 0.00 \\
\hline & & Weighted Median & 0.72 & 0.58 & 0.90 & 0.00 \\
\hline & & MR-PRESSO & 0.74 & 0.61 & 0.90 & 0.00 \\
\hline & & MR Egger slope & 0.47 & 0.24 & 0.92 & 0.03 \\
\hline & & MR Egger intercept $_{\text {t }}$ & $(0.00)$ & -0.00 & 0.01 & 0.16 \\
\hline
\end{tabular}

*Estimates are presented as ORs for causal effect estimates and $\beta$-values for the MR Egger intercept, which is a measure of directional pleiotropy. The unit for neuroticism was per standard deviation increase. ${ }^{\dagger}$ Outlier rs58879558 removed. MR, Mendelian randomization; SNP, single nucleotide polymorphism; OR, odds ratio; CI, confidence interval; PD, Parkinson's disease; IVW, inverse variance weighting.

129 SNPs (all)

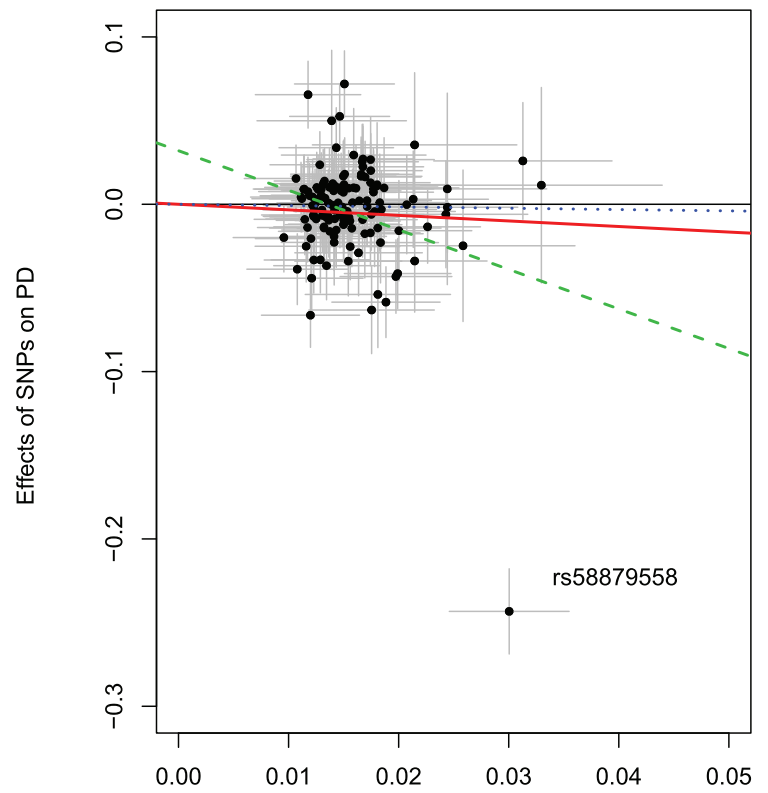

128 SNPs (outlier removed)

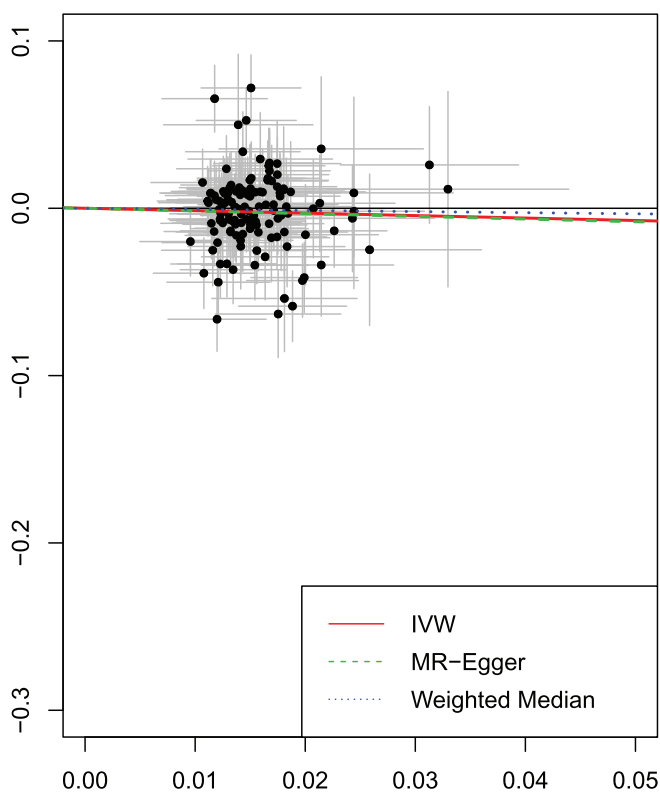

Effects of SNPs on Neuroticism

Fig. 2. Scatter plots showing the effect estimates (with 95\% confidence intervals) of SNP-neuroticism associations and SNP-PD risk associations, with (left panel) and without (right panel) outlier. Lines represent the three Mendelian randomization estimates. IVW, inverse variance weighted method; SNP, single nucleotide polymorphism 

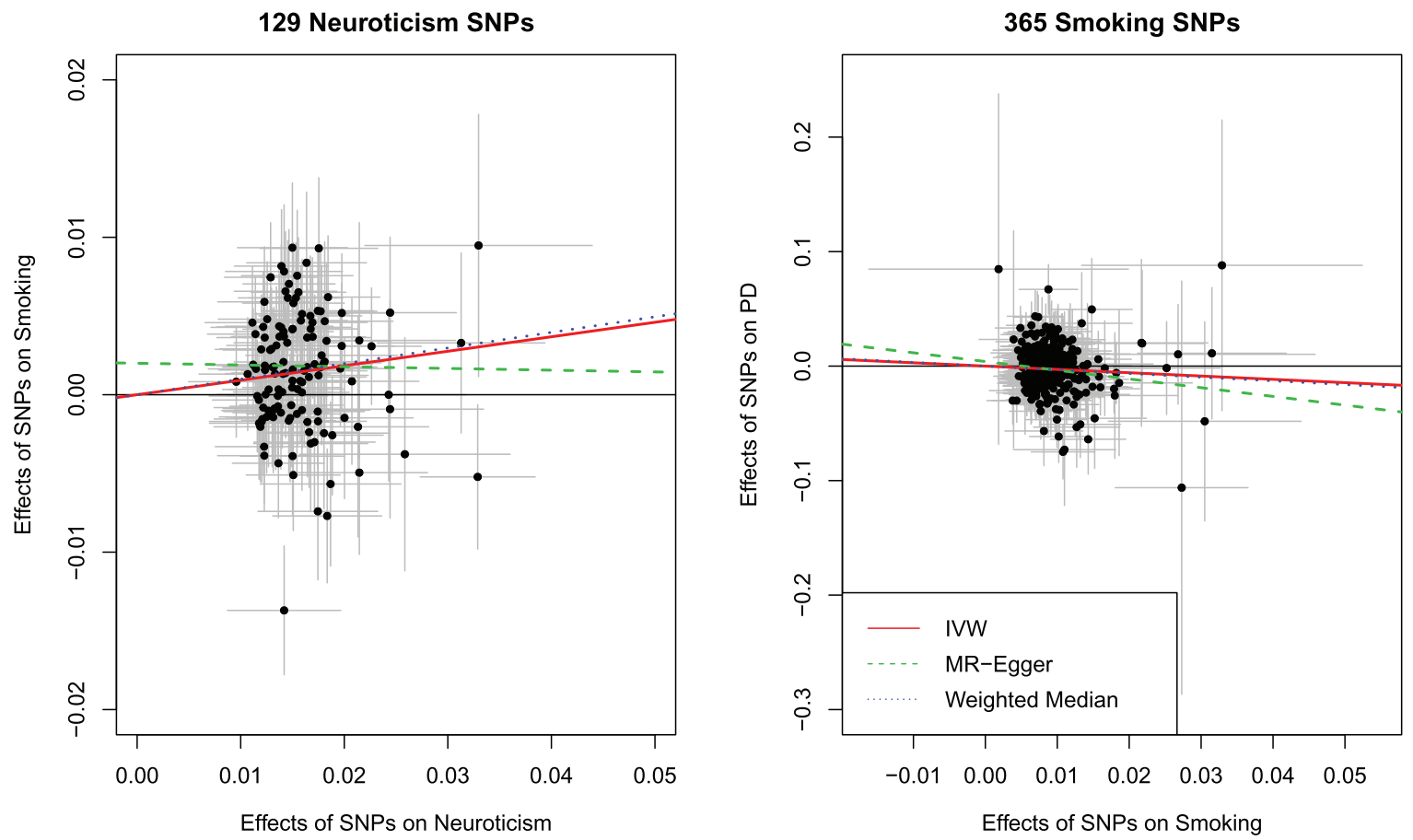

Fig. 3. Scatter plot showing the effect estimates (with 95\% confidence intervals) of SNP-neuroticism associations and SNP-Smoking Initiation associations for all 129 Neuroticism SNPs (left panel). Scatter plot showing the effect estimates (with 95\% confidence intervals) of SNP-smoking initiation associations and SNP-PD risk associations for all 365 smoking initiation SNPs (right panel). Lines represent the three Mendelian randomization estimates. IVW, inverse variance weighted method; SNP, single nucleotide polymorphism; PD, Parkinson's disease; OR, odds ratio; $\mathrm{CI}$, confidence interval.

that the effect was not substantial. No strong evidence was obtained for horizontal pleiotropy (Intercept from the MR-Egger regression: $0.00,95 \% \mathrm{CI}:-0.00$ to 0.01 ). No important outliers were detected in the scatter plot of effect estimates on neuroticism and smoking initiation for each SNP (Fig. 3, left panel). The funnel plot showed that the causal effect estimates for each SNP were symmetrically spread around the IVW and weighted median estimate, indicating that these effect estimates were not influenced by directional pleiotropy. Conversely, the MR-Egger estimate might have been affected by single influential SNPs explaining the lack of an effect observed using the MR-Egger approach (Supplementary Figure 4). No influential outlier was detected in the leave-one-out analysis (Supplementary Figure 5).

\section{Smoking initiation and PD risk}

Among the 378 SNPs associated with smoking initiation, 74 were unavailable in the PD GWAS and/or palindromic. We were able to identify 61 proxy SNPs leaving 365 SNPs for analyses (Supplementary
Tables 1 and 3). Using the IVW method, we found a strong association between smoking initiation and a lower risk of PD (OR: $0.75,95 \%$ CI: 0.62 to 0.91 ). Similar results were obtained from the weighted median method (OR: $0.72,95 \%$ CI: 0.58 to 0.90 ) and MR Egger regression (OR: 0.47, 95\% CI: 0.24 to 0.92 ). There was evidence for heterogeneity among the SNPs in the causal effect of smoking initiation on PD risk ( $p$-value $=0.008)$, although the $I^{2}$ of $15.5 \%$ indicated that the amount of heterogeneity was not substantial. Further, there was no strong evidence of horizontal pleiotropy according to the MR Egger intercept ( $\beta 0.00,95 \%$ CI: -0.00 to 0.01 ). No important outliers were detected in the scatter plot of effect estimates on smoking initiation and PD risk for each SNP (Fig. 3, right panel). Further, the funnel plot showed that the causal effect estimates for each SNP were symmetrically spread around the overall effect estimates using the IVW, weighted median estimate, and the MR-Egger method indicating that the results were not influenced by directional pleiotropy and no influential outlier SNP was observed (Supplementary Figures 6 and 7). 


\section{Sensitivity analysis}

As a sensitivity analysis, we repeated all analyses excluding proxy SNPs, and the results largely corroborated the results presented above (Supplementary Table 4). We also analyzed the effects of neuroticism and smoking initiation on PD risk separately depending on whether the PD diagnosis was based on self-report $(n=9,157)$ or clinical diagnosis $(n=27,595)$. The results from these analyses (Supplementary Table 5) were comparable to the main analyses presented in Table 1 .

\section{DISCUSSION}

In this study, we used two-sample MR methods to disentangle the causal associations among neuroticism, smoking, and PD using GWAS summary statistics curated from the latest meta-analyses of large cohorts of European ancestry. While we did not find a significant association between neuroticism and PD, one SNP, rs58879558 (located in MAPT region), was associated with both neuroticism and PD. Further, we found evidence supporting a causal association between neuroticism and smoking initiation and smoking initiation was again associated with reduced risk of $\mathrm{PD}$.

\section{Interpretations in the context of previous research}

Neuroticism has previously been associated with subsequent increased PD risk after adjustment for age, sex, smoking, education, and frequency of hospital visits. Further, smoking was identified as a significant mediator that suppressed this association [4]. Thus, we used a network MR design to estimate the effect of neuroticism on PD risk, as well as to assess the role of smoking in this relationship. Although no evidence was found in support of an effect of neuroticism on PD risk in our MR analysis as reported in observational studies [4, 5], significant associations were observed between neuroticism and smoking initiation, and between smoking initiation and PD risk. Our MR analyses of smoking initiation and the risk of PD have a considerable overlap with previous MR studies on this topic, where smoking (ever smoker) was associated with a lower risk of PD $[9,30]$. Taken together, the results are in line with observational evidence of an indirect path between neuroticism and PD risk through smoking.

In addition to neuroticism, other personality traits that has been investigated in relation to PD include openness, extraversion, novelty seeking, harm avoidance, reward dependence, perseverance/persistence [31-43]. A meta-analysis [44] summarizing these results found that both higher levels of neuroticism and lower levels of openness and extraversion were associated with higher risks of PD. While reward dependence and persistence showed no significant results, lower levels of novelty seeking and higher levels of harm avoidance were also associated with risks of PD. These studies, taken together, highlighted the complicated features of personality in the early diagnosis and prognosis of PD.

Our finding that the association between neuroticism and PD may be due to shared genetics is consistent with a previous study reporting that neuroticism and PD have some overlapping genes [8]. When excluding this variant in the MR analysis, the magnitude of the association was considerably attenuated, implying that genetics play an important role in the observed associations in conventional epidemiological studies and the variant may exhibit pleiotropic effects in MR analysis. It is therefore crucial to take genetic confounding into account when examining the role of neuroticism in PD.

We found new evidence to support a causal role of neuroticism in smoking initiation, which is in line with published observational cohort studies [10]. A previous MR study using ten SNPs as instrumental variables for neuroticism reported that neuroticism was associated with increased odds ratio for smoking initiation although with a wide confidence interval and statistically not significant [12]. Our study, empowered with more SNPs as instrumental variables and a larger sample size for smoking initiation, updates previous findings and provides additional evidence for the causal association between them. These results could be informative for future implementation of smoking intervention strategies.

We found that smoking initiation was associated with a reduced risk of PD. This finding is consistent with previous MR studies that reported a reduced risk of PD for ever smokers [30] and for smoking initiation [9]. Our findings are also in line with numerous observational studies consistently showing a reduced risk of PD in relation to smoking [13]. Several explanations have been suggested for this association, including residual confounding and reverse causation such that individuals with prodromal PD are more likely to quit smoking [18]. However, some findings from observational studies support a causal effect of smoking on PD. For example, smoking intensity and duration are both related to PD risk reduction [14], 
and passive smoking among never smokers has also been associated with reduced PD risk [17]. Further, smoking has also been associated with reduced PD risk in co-twin control studies, reducing confounding due to genetic and familial environmental factors [45]. Taken together, these findings support the theory that smoking has a protective effect on PD risk.

\section{Strengths and limitations of the study}

A main strength of this two-sample MR study is the large sample size accrued from GWAS summary statistics that provided high statistical power to examine causal relationships between neuroticism, smoking initiation, and PD. Another advantage is that the use of genetic variants as a proxy for an exposure may be a better measure of life-time exposure levels than single measurements. For example, results from personality tests could be affected by periodic fluctuations or stochastic influences on personality.

The limitations of this study mainly concern the general assumptions of MR analysis. The first assumption that the SNPs used as instrumental variables must be associated with the exposure is the only testable assumption. We only used SNPs that were associated with the exposures at genome-wide significance which makes this assumption more likely to hold. The second assumption is that the SNPs should not be associated with any confounding factors between neuroticism, smoking initiation and PD. MR studies are less vulnerable to confounding compared to observational studies given the random assortment of alleles. However, violation of this assumption is possible and cannot be ruled out. One important potential cause of violation to this assumption is population stratification. However, we only included data based on participants of European ancestry reducing this potential bias. The third assumption is that the SNPs should only affect the outcome through the exposure, i.e., there should be no pleiotropy. We performed several tests to examine for potential pleiotropic effects and the SNPs that violated this MR assumption was removed. The Cochran's Q test of heterogeneity was overall significant; this may however not be a major concern, given that the $\mathrm{I}^{2}$ statistic was generally low, the MR Egger intercept was overall non-significant in our main analyses, and the Cochran's Q test is known to be overpowered when using many SNPs as in this study. Moreover, we used different MR models that allow for different types of genetic pleiotropy and make different assumptions regarding instrument validity. As these different models produced similar causal effect estimates, our findings seem to be robust. In addition, because of the non-significant findings of the association between neuroticism and PD, our analysis is unable to estimate whether there is a direct effect and to assess whether this direct effect may be in the opposite direction compared to the direct effect.

\section{CONCLUSION}

In this study, we found no evidence that genetic liability to neuroticism is associated with increased risk of PD, but with an increased tendency to start smoking regularly. Further, our study provided evidence that genetic liability to smoking initiation is associated with a reduced risk of PD. Future studies with large sample sizes containing information on both smoking phenotypes and PD in the same dataset with the potential to stratify on smoking status, may investigate whether smoking heaviness or cessation are also causally related to PD risk, to increase the understanding of the relationship between smoking and PD.

\section{ACKNOWLEDGMENTS}

We thank the employees and research participants of 23andMe for the use of the GWAS summary statistics, Dr. Dylan Williams for his assistance in data access, and IPDGC for providing GWAS summary statistics.

This study was funded by the Swedish Research Council (grant number: 2017-02175). The funder has no role in study design, analysis, or writing.

\section{CONFLICT OF INTEREST}

The authors have no conflict of interest to report.

\section{SUPPLEMENTARY MATERIAL}

The supplementary material is available in the electronic version of this article: https://dx.doi.org/ 10.3233/JPD-202522.

\section{REFERENCES}

[1] Kalia LV, Lang AE (2015) Parkinson's disease. Lancet 386, 896-912.

[2] Santangelo G, Piscopo F, Barone P, Vitale C (2017) Personality in Parkinson's disease: Clinical, behavioural and cognitive correlates. J Neurol Sci 374, 17-25. 
[3] Ishihara L, Brayne C (2006) What is the evidence for a premorbid parkinsonian personality: A systematic review. Mov Disord 21, 1066-1072.

[4] Sieurin J, Gustavsson P, Weibull CE, Feldman AL, Petzinger GM, Gatz M, Pedersen NL, Wirdefeldt K (2016) Personality traits and the risk for Parkinson disease: A prospective study. Eur J Epidemiol 31, 169-175.

[5] Bower JH, Grossardt BR, Maraganore DM, Ahlskog JE, Colligan RC, Geda YE, Therneau TM, Rocca WA (2010) Anxious personality predicts an increased risk of Parkinson's disease. Mov Disord 25, 2105-2113.

[6] Laceulle OM, Ormel J, Aggen SH, Neale MC, Kendler KS (2013) Genetic and environmental influences on the longitudinal structure of neuroticism: A trait-state approach. Psychol Sci 24, 1780-1790.

[7] Vukasovic T, Bratko D (2015) Heritability of personality: A meta-analysis of behavior genetic studies. Psychol Bull 141, 769-785.

[8] Nagel M, Jansen PR, Stringer S, Watanabe K, de Leeuw CA, Bryois J, Savage JE, Hammerschlag AR, Skene NG, Muñoz-Manchado AB; 23andMe Research Team, White $\mathrm{T}$, Tiemeier H, Linnarsson S, Hjerling-Leffler J, Polderman TJC, Sullivan PF, van der Sluis S, Posthuma D (2018) Meta-analysis of genome-wide association studies for neuroticism in 449,484 individuals identifies novel genetic loci and pathways. Nat Genet 50, 920-927.

[9] Nalls MA, Blauwendraat C, Vallerga CL, Heilbron K, Bandres-Ciga S, Chang D, Tan M, Kia DA, Noyce AJ, Xue A, Bras J, Young E, von Coelln R, Simón-Sánchez J, Schulte C, Sharma M, Krohn L, Pihlstrøm L, Siitonen A, Iwaki H, Leonard H, Faghri F, Gibbs JR, Hernandez DG, Scholz SW, Botia JA, Martinez M, Corvol JC, Lesage S, Jankovic J, Shulman LM, Sutherland M, Tienari P, Majamaa K, Toft M, Andreassen OA, Bangale T, Brice A, Yang J, Gan-Or Z, Gasser T, Heutink P, Shulman JM, Wood NW, Hinds DA, Hardy JA, Morris HR, Gratten J, Visscher PM, Graham RR, Singleton AB; 23andMe Research Team; System Genomics of Parkinson's Disease Consortium; International Parkinson's Disease Genomics Consortium (2019) Identification of novel risk loci, causal insights, and heritable risk for Parkinson's disease: A meta-analysis of genome-wide association studies. Lancet Neurol 18, 1091-1102.

[10] Munafo MR, Zetteler JI, Clark TG (2007) Personality and smoking status: A meta-analysis. Nicotine Tob Res $\mathbf{9}$, 405-413.

[11] Hakulinen C, Hintsanen M, Munafo MR, Virtanen M, Kivimaki M, Batty GD, Jokela M (2015) Personality and smoking: Individual-participant meta-analysis of nine cohort studies. Addiction 110, 1844-1852.

[12] Sallis HM, Davey Smith G, Munafo MR (2019) Cigarette smoking and personality: Interrogating causality using Mendelian randomisation. Psychol Med 49, 2197-2205.

[13] Li X, Li W, Liu G, Shen X, Tang Y (2015) Association between cigarette smoking and Parkinson's disease: A metaanalysis. Arch Gerontol Geriatr 61, 510-516.

[14] Chen H, Huang X, Guo X, Mailman RB, Park Y, Kamel F, Umbach DM, Xu Q, Hollenbeck A, Schatzkin A, Blair A (2010) Smoking duration, intensity, and risk of Parkinson disease. Neurology 74, 878-884.

[15] Ritz B, Ascherio A, Checkoway H, Marder KS, Nelson LM, Rocca WA, Ross GW, Strickland D, Van Den Eeden SK, Gorell J (2007) Pooled analysis of tobacco use and risk of Parkinson disease. Arch Neurol 64, 990-997.

[16] Gallo V, Vineis P, Cancellieri M, Chiodini P, Barker RA, Brayne C, Pearce N, Vermeulen R, Panico S, Bueno-de-
Mesquita B, Vanacore N, Forsgren L, Ramat S, Ardanaz E, Arriola L, Peterson J, Hansson O, Gavrila D, Sacerdote C, Sieri S, Kuhn T, Katzke VA, van der Schouw YT, Kyrozis A, Masala G, Mattiello A, Perneczky R, Middleton L, Saracci R, Riboli E (2019) Exploring causality of the association between smoking and Parkinson's disease. Int J Epidemiol 48, 912-925.

[17] Searles Nielsen S, Gallagher LG, Lundin JI, Longstreth WT, Jr., Smith-Weller T, Franklin GM, Swanson PD, Checkoway H (2012) Environmental tobacco smoke and Parkinson's disease. Mov Disord 27, 293-296.

[18] Ritz B, Lee PC, Lassen CF, Arah OA (2014) Parkinson disease and smoking revisited: Ease of quitting is an early sign of the disease. Neurology 83, 1396-1402.

[19] Chuang YH, Paul KC, Sinsheimer JS, Bronstein JM, Bordelon YM, Ritz B (2019) Genetic variants in nicotinic receptors and smoking cessation in Parkinson's disease. Parkinsonism Relat Disord 62, 57-61.

[20] Postuma RB, Aarsland D, Barone P, Burn DJ, Hawkes CH, Oertel W, Ziemssen T (2012) Identifying prodromal Parkinson's disease: Pre-motor disorders in Parkinson's disease. Mov Disord 27, 617-626.

[21] Bycroft C, Freeman C, Petkova D, Band G, Elliott LT, Sharp K, Motyer A, Vukcevic D, Delaneau O, O'Connell J, Cortes A, Welsh S, Young A, Effingham M, McVean G, Leslie S, Allen N, Donnelly P, Marchini J (2018) The UK Biobank resource with deep phenotyping and genomic data. Nature 562, 203-209.

[22] Eriksson N, Macpherson JM, Tung JY, Hon LS, Naughton B, Saxonov S, Avey L, Wojcicki A, Pe'er I, Mountain J (2010) Web-based, participant-driven studies yield novel genetic associations for common traits. PLoS Genet 6, e1000993.

[23] de Moor MH, Costa PT, Terracciano A, Krueger RF, de Geus EJ, Toshiko T, Penninx BW, Esko T, Madden PA, Derringer J, Amin N, Willemsen G, Hottenga JJ, Distel MA, Uda M, Sanna S, Spinhoven P, Hartman CA, Sullivan P, Realo A, Allik J, Heath AC, Pergadia ML, Agrawal A, Lin P, Grucza R, Nutile T, Ciullo M, Rujescu D, Giegling I, Konte B, Widen E, Cousminer DL, Eriksson JG, Palotie A, Peltonen L, Luciano M, Tenesa A, Davies G, Lopez LM, Hansell NK, Medland SE, Ferrucci L, Schlessinger D, Montgomery GW, Wright MJ, Aulchenko YS, Janssens AC, Oostra BA, Metspalu A, Abecasis GR, Deary IJ, Raikkonen K, Bierut LJ, Martin NG, van Duijn CM, Boomsma DI (2012) Metaanalysis of genome-wide association studies for personality. Mol Psychiatry 17, 337-349.

[24] Liu M, Jiang Y, Wedow R, Li Y, Brazel DM, Chen F, Datta G, Davila-Velderrain J, McGuire D, Tian C, Zhan X; 23andMe Research Team; HUNT All-In Psychiatry, Choquet H, Docherty AR, Faul JD, Foerster JR, Fritsche LG, Gabrielsen ME, Gordon SD, Haessler J, Hottenga JJ, Huang H, Jang SK, Jansen PR, Ling Y, Mägi R, Matoba N, McMahon G, Mulas A, Orrù V, Palviainen T, Pandit A, Reginsson GW, Skogholt AH, Smith JA, Taylor AE, Turman C, Willemsen G, Young H, Young KA, Zajac GJM, Zhao W, Zhou W, Bjornsdottir G, Boardman JD, Boehnke M, Boomsma DI, Chen C, Cucca F, Davies GE, Eaton CB, Ehringer MA, Esko T, Fiorillo E, Gillespie NA, Gudbjartsson DF, Haller T, Harris KM, Heath AC, Hewitt JK, Hickie IB, Hokanson JE, Hopfer CJ, Hunter DJ, Iacono WG, Johnson EO, Kamatani Y, Kardia SLR, Keller MC, Kellis M, Kooperberg C, Kraft P, Krauter KS, Laakso M, Lind PA, Loukola A, Lutz SM, Madden PAF, Martin NG, McGue M, McQueen MB, Medland SE, Metspalu A, Mohlke KL, Nielsen JB, Okada Y, Peters U, Polderman TJC, Posthuma 
D, Reiner AP, Rice JP, Rimm E, Rose RJ, Runarsdottir V, Stallings MC, Stančáková A, Stefansson H, Thai KK, Tindle HA, Tyrfingsson T, Wall TL, Weir DR, Weisner C, Whitfield JB, Winsvold BS, Yin J, Zuccolo L, Bierut LJ, Hveem K, Lee JJ, Munafò MR, Saccone NL, Willer CJ, Cornelis MC, David SP, Hinds DA, Jorgenson E, Kaprio J, Stitzel JA, Stefansson K, Thorgeirsson TE, Abecasis G, Liu DJ, Vrieze S (2019) Association studies of up to 1.2 million individuals yield new insights into the genetic etiology of tobacco and alcohol use. Nat Genet 51, 237-244.

[25] Machiela MJ, Chanock SJ (2015) LDlink: A web-based application for exploring population-specific haplotype structure and linking correlated alleles of possible functional variants. Bioinformatics 31, 3555-3557.

[26] Burgess S, Scott RA, Timpson NJ, Davey Smith G, Thompson SG, EPIC- InterAct Consortium (2015) Using published data in Mendelian randomization: A blueprint for efficient identification of causal risk factors. Eur J Epidemiol 30, 543-552.

[27] Bowden J, Davey Smith G, Burgess S (2015) Mendelian randomization with invalid instruments: Effect estimation and bias detection through Egger regression. Int J Epidemiol 44, 512-525.

[28] Bowden J, Davey Smith G, Haycock PC, Burgess S (2016) Consistent estimation in Mendelian randomization with some invalid instruments using a weighted median estimator. Genet Epidemiol 40, 304-314.

[29] Yavorska OO, Burgess S (2017) MendelianRandomization: An R package for performing Mendelian randomization analyses using summarized data. Int J Epidemiol 46, 17341739.

[30] Grover S, Lill CM, Kasten M, Klein C, Del Greco MF, Konig IR (2019) Risky behaviors and Parkinson disease: A mendelian randomization study. Neurology 93, e1412e1424.

[31] Baig F, Lawton MA, Rolinski M, Ruffmann C, Klein JC, Nithi K, Okai D, Ben-Shlomo Y, Hu MT (2017) Personality and addictive behaviours in early Parkinson's disease and REM sleep behaviour disorder. Parkinsonism Relat Disord 37, 72-78.

[32] Farnikova K, Obereigneru R, Kanovsky P, Prasko J (2012) Comparison of personality characteristics in Parkinson disease patients with and without impulse control disorders and in healthy volunteers. Cogn Behav Neurol 25, 25-33.

[33] Volpato C, Signorini M, Meneghello F, Semenza C (2009) Cognitive and personality features in Parkinson disease: 2 sides of the same coin? Cogn Behav Neurol 22, 258-263.
[34] Tomer R, Aharon-Peretz J (2004) Novelty seeking and harm avoidance in Parkinson's disease: Effects of asymmetric dopamine deficiency. J Neurol Neurosurg Psychiatry 75, 972-975.

[35] Fujii C, Harada S, Ohkoshi N, Hayashi A, Yoshizawa K (2000) Cross-cultural traits for personality of patients with Parkinson's disease in Japan. Am J Med Genet 96, 1-3.

[36] Jacobs H, Heberlein I, Vieregge A, Vieregge P (2001) Personality traits in young patients with Parkinson's disease. Acta Neurol Scand 103, 82-87.

[37] Kaasinen V, Nurmi E, Bergman J, Eskola O, Solin O, Sonninen P, Rinne JO (2001) Personality traits and brain dopaminergic function in Parkinson's disease. Proc Natl Acad Sci U S A 98, 13272-13277.

[38] Menza MA, Forman NE, Goldstein HS, Golbe LI (1990) Parkinson's disease, personality, and dopamine. J Neuropsychiatry Clin Neurosci 2, 282-287.

[39] McNamara P, Durso R, Harris E (2007) "Machiavellianism" and frontal dysfunction: Evidence from Parkinson's disease. Cogn Neuropsychiatry 12, 285-300.

[40] Bodi N, Keri S, Nagy H, Moustafa A, Myers CE, Daw N, Dibo G, Takats A, Bereczki D, Gluck MA (2009) Rewardlearning and the novelty-seeking personality: A betweenand within-subjects study of the effects of dopamine agonists on young Parkinson's patients. Brain 132, 2385-2395.

[41] Fassino S, Abbate Daga G, Gramaglia C, Piero A, Zibetti M, Castelli L, Cinquepalmi A, La Notte M, Lopiano L (2010) Novelty-seeking in Parkinson's disease after deep brain stimulation of the subthalamic nucleus: A case-control study. Psychosomatics 51, 62-67.

[42] Diaz-Santos M, Cao B, Yazdanbakhsh A, Norton DJ, Neargarder S, Cronin-Golomb A (2015) Perceptual, cognitive, and personality rigidity in Parkinson's disease. Neuropsychologia 69, 183-193.

[43] Evans AH, Lawrence AD, Potts J, MacGregor L, Katzenschlager R, Shaw K, Zijlmans J, Lees AJ (2006) Relationship between impulsive sensation seeking traits, smoking, alcohol and caffeine intake, and Parkinson's disease. J Neurol Neurosurg Psychiatry 77, 317-321.

[44] Santangelo G, Garramone F, Baiano C, D'Iorio A, Piscopo F, Raimo S, Vitale C (2018) Personality and Parkinson's disease: A meta-analysis. Parkinsonism Relat Disord 49, 67-74.

[45] Wirdefeldt K, Gatz M, Pawitan Y, Pedersen NL (2005) Risk and protective factors for Parkinson's disease: A study in Swedish twins. Ann Neurol 57, 27-33. 